\title{
EAPS8-1169 Diabetic Ketoacidosis at oncet of type 1 diabetes (study of 173 cases)
}

Kmiha S, Ammous M, Chabchoub I, Benali H, Kamoun Th, Aloulou H, Hachicha M Department of Pediatrics, Hedi Chaker University Hospital of Sfax, TUNISIA

Background: Diabetic Ketoacidosis (DKA) at oncet of type 1 diabetes (T1D) is still a fairly common finding, particularly in young children, and is the leading cause of morbidity and mortality.

Aim: To study the epidemiological, clinical, biological, immunological, therapeutic and evolutive features of DKA at oncet of T1D in order to better understand the factors associated with its severity.

Methods: A retrospective, descriptive and comparative study was conducted among children who were hospitalized for DKA at oncet of T1D between January 2000 and December 2015 at the Pediatric Department of Hedi Chaker University Hospital of Sfax, Tunisia.

Results: We collected 173 new cases of type 1 diabetic children admitted for DKA at oncet of T1D; the hospital frequency was 28 per 10000 hospitalizations with an average annual incidence of 11 new cases per year. During the study period, DKA revealed diabetes in 173 of 450 new T1D cases (38.4\%). We divided the cases into 3 groups: group 1 (mild DKA: 36 children (20.8\%)); group 2 (moderately severe DKA: 55 children $(31.79 \%))$ and group 3 (severe DKA: 82 children (47.4\%)). The mean age of the children was 7.13 years \pm 3.98 . There were 81 boys $(46.82 \%)$ and 92 girls $(53.18 \%)$ (sex ratio: $0.88)$. There were more children with familial T1D in group $1(25 \%)$ and group $2(21.8 \%)$ than in group $3(13.4 \%)(p=0.24)$. Polyuria and polydipsia were reported in $99.4 \%$ of cases, its duration was not correlated with the severity of the DKA $(p=0.079)$. At admission, $20.2 \%$ of children had an altered level of consciousness. The mean venous glucose was $26.17 \mathrm{mmol} / \mathrm{I} \pm 6.91$. Hypokalemia was more common in group 3 ( $p$ $<0.001$ ). Among our children, 127 children were treated according to the Lestradet protocol and 34 children were treated according to the ISPAD protocol. The decrease in capillary as well as venous glucose, the decrease in ketonuria and the increase in $\mathrm{pH}$ up to $\mathrm{H} 6$ of the protocol were significantly greater in children treated according to the ISPAD protocol compared to those treated according to the Lestradet protocol. The linear regression study showed that during treatment, the ISPAD protocol was more contributive to decrease blood glucose levels, to increase $\mathrm{pH}$ and to correct sodium and potassium levels; compared to the Lestradet protocol. The most common complication in the acute phase was hypokalemia (80.12\%). After stopping the IV insulin therapy protocol, all children were put on a conventional insulin therapy regimen with 2 injections at an average dose of $0.85 \mathrm{lU} / \mathrm{kg} /$ day \pm 0.23 . The mean follow-up was 4.07 years \pm 3.15. We noted 2 deaths.

Conclusion: DKA at oncet of T1D is the leading cause of death in children with T1D. The place of prevention is decisive; this requires appropriate screening of children at high risk for T1D and information for parents and health workers about the first clinical signs of diabetes mellitus. 